\title{
The Tripartite Intervention: Breaking Down Attitudinal Barriers in Education
}

\author{
John R. R. Freer \\ University of Windsor \& St. Clair College \\ Faculty of Education, University of Windsor, Windsor, ON, Canada \\ School of Community Studies, St. Clair College, Windsor, ON, Canada \\ jfreer@uwindsor.ca \\ jfreer@stclaircollege.ca
}

\begin{abstract}
Students with disabilities face attitudinal barriers to social inclusion. Poor attitudes toward disability can impact friendship development between students with and without disabilities and result in social exclusion. Fortunately, a body of literature exists, which suggests that educational interventions can help to enhance students' attitudes toward disability. Such interventions, however, vary in both their approach to teaching students about disability experiences and their effectiveness. This paper presents the Tripartite Intervention, a 12-lesson intervention created for junior-level elementary students that targets cognitive, behavioural, and affective dimensions of attitude. This paper provides a high-level overview of the intervention lessons, as well as, offers readers some practical considerations for implementation. Those interested in utilizing the Tripartite Intervention for educational or research purposes are encouraged to contact the author for intervention materials and training opportunities.
\end{abstract}

\section{Keywords}

disability - attitude - ableism - educational intervention - lesson materials

\section{Introduction}

The field of disability studies has revolutionized the way in which disability is viewed. Specifically, the social model of disability has helped people to 
recognize that disability is, at least in part, a social construction and that people with disabilities continue to face barriers to access and inclusion in society (Jones 1996; Oliver 1996). The World Health Organization (2020) defines disability as a 'complex phenomenon, reflecting the interaction between features of a person's body and features of the society in which he or she lives' (para. 2). Disability Studies in Education (DSE) scholars have pointed out that students with disabilities face barriers in educational contexts (Baglieri et al. 2011; Slee, Corcoran, \& Best 2019). Barriers can come in many forms (e.g., physical, communication, etc.). Attitudinal barriers pose a unique challenge because they are often invisible and can perpetuate ableism.

Ableism is a complex system of expectations about ability and can include stereotypes, prejudice, and discrimination toward people with disabilities. These (often low) expectations can impact the experiences that people with disabilities have in society, including within educational institutions. For example, it is not uncommon for individuals to express pity toward people with disabilities (Shapiro 1994). Hehir (2002) further explains that 'ableist assumptions in the education of children with disabilities not only reinforces prevailing prejudices against disability but may very well contribute to low levels of educational attainment and employment' (p. 4). Ableism can take many forms. Storey (2007) suggests that ableism occurs at individual, institutional, and cultural levels. At the individual level, poor attitudes toward disability can present barriers for students who have disabilities.

In an educational context many stakeholders' attitudes toward disability can impact students' experiences (de Boer et al. 2012). Peers' attitudes are perhaps the most important when it comes to social inclusion because a major aspect of social inclusion is interpersonal relationships (Simplican, Leader, \& Kosciulek 2015). Students' attitudes toward disability matter a great deal because attitudes can presage behaviour, which gives children the power to include or exclude their peers with disabilities. Children develop attitudes toward disability at young ages and this can play a role in formation of students' in- and out-groups. If left unexamined, negative attitudes could persist into adulthood. Many factors impact the development of students' attitudes toward disability (e.g., experience with disability, role model's attitudes, etc.; de Boer, Pijl, \& Minnaert 2012). After conducting a meta-analysis, Nowicki and Sandieson (2002) concluded, 'in general, children's attitudes towards persons with disabilities are in need of improvement' (p. 263).

Despite efforts for more inclusive education, students with disabilities continue to report fewer friendships with their same-age peers (Avramidis 2013; Koster et al. 2010; Petry 2018). Some believe this social inequity is a result of attitudinal barriers or ableism (de Boer et al., 2013; Nowicki \& Sandieson 2002; 
Vignes et al. 2009). In the literature, there has been a call to address ableism in schools. Fortunately, researchers in this field of study have also discovered that educational interventions are generally effective in enhancing students' attitudes toward disability (Freer 2020; Lindsay \& Edwards 2013). In fact, these interventions have the potential to have lasting effects. One research team found that changes in students' attitudes were present eight years after the administration of their intervention (Rillotta \& Nettelbeck 2007). Due to the well documented effectiveness of attitudinal interventions, many different approaches have been developed (e.g., awareness training, embodied experiences, etc.).

Researchers involved in developing anti-ableist interventions have to make important decisions regarding the measurement of attitude. Typically, students' attitudes toward disability are measured before and after an intervention to determine its effectiveness. When selecting an attitude scale, researchers also need to consider how they are defining attitude. Many theories of attitude exist (e.g., the Tripartite Theory, the Affect Primary Hypothesis, the Expectancy-Value Model, etc.). How a researcher operationalizes attitude can impact the selection of a measurement tool. For example, if one subscribes to a unidimensional theory of attitude, a unidimensional scale ought to be used to measure attitude. Among researchers interested in measuring students' attitudes toward disability, the Tripartite Theory of attitude continues to be sought after in attitude metrics for children (Vignes et al. 2008). Proponents of the Tripartite Theory view attitude as equal parts affect, behaviour, and cognition. This theory can be a powerful way to examine prejudice (affect), discrimination (behaviour), and stereotypes (cognition; Jackson et al. 1996). Many researchers select attitude metrics with subscales for each of these three dimensions of attitude, such as the Chedoke-McMaster Attitudes toward Children with Handicaps (САTCH) scale (Rosenbaum, Armstrong, \& King 1986). The CATCH scale was developed in Ontario, Canada for students in Grades 4 to 8.

Many intervention studies in this field of research lack intervention and metric congruency. Intervention and metric congruency occurs when an intervention explicitly targets the same dimensions of attitude being measured by the selected attitude metric. Despite the widespread use of tripartite measures of attitude, most intervention programs aimed at enhancing students' attitudes toward disability reported in the literature do not explicitly target all three dimensions of attitude (i.e., feelings, thoughts, and actions). Many researchers only target one (Godeau et al. 2010; Lloyd et al. 2017; Tavares 2011) or two dimensions of attitude (e.g., Giagazoglou \& Papadaniil 2018; Krahé \& Altwasser 2006). Rillotta and Nettelbeck (2007) were an exception to the rule. 
They utilized all three dimensions of attitude in their intervention but did not explicitly discuss the Tripartite Theory in their article.

To address this gap, the Tripartite Intervention (ті) was developed with the intention of targeting cognitive, affective, and behavioural components of students' attitudes. The TI is a 12-lesson intervention aimed at enhancing students' attitudes toward disability to help counter ableism in elementary schools. There is an extensive body of literature on ableism in schools and efforts aimed at enhancing students' attitudes toward disability (e.g., Connor \& Bejoian 2007; Lindsay \& Edwards 2013; Storey 2007).

The initial implementation of the TI resulted in significantly more positive scores on the cognitive dimension of the СATCH scale for students in the experimental group (Freer 2020). Rather than discuss the findings from the initial implementation of the $\mathrm{TI}$ in this article, the TI is described in detail for teachers and researchers who might be interested in utilizing these lessons in their classes and/or research projects. If readers would like copies of the intervention materials (e.g., lesson plans, slideshows, worksheets, video links, etc.) and training on the Ti for educational or research purposes, they are encouraged to email the author at jfreer@uwindsor.ca or jfreer@stclaircollege. ca. The TI lessons include: learning outcomes, an agenda with timed activities, teacher's resources (e.g., links to additional info), teacher's notes (e.g., talking points for discussion), required materials, strategies for differentiated instruction, and strategies for classroom management. To follow will be a detailed description of the ті lessons, as well as some practical considerations for those looking to implement this intervention.

\section{Intervention Development}

The intervention lessons were created for junior education in Ontario, Canada. Students in junior education (i.e., Grades 4 to 6 ) are typically between 8 and 12-years of age. Researchers have suggested that students around this age may have difficulty understanding disability, especially beyond physical disability (Louari 2013; Nowicki 2007). Junior education may be an ideal time to teach students about disability, so they can develop greater understandings and challenge preconceived notions about disability (e.g., ableist attitudes). Students typically have a better understanding of physical and sensory disabilities compared to less obvious exceptionalities (e.g., learning disabilities; de Boer, Pijl, \& Minnaert 2012; Freer, 2020). Many of the lessons focus on the types of disabilities students are likely to be familiar with, but there are elements of the TI that provide students with an opportunity to consider diverse disability experiences. 
All three dimensions of attitude were targeted in the lessons, based on successful strategies found in existing literature (e.g., Connor \& Bejoian 2007; Krahé \& Altwasser 2006; Macmillan et al. 2014; Tindall 2013). The exception to this was the affective-based lessons because there was a scarcity of interventions that targeted this dimension explicitly. For the affective lessons, research outside the field (e.g., psychological theories of emotion) were consulted. Each lesson takes approximately 45 to 75 minutes to administer. The intervention consists of two six-lesson units about disability, for a total of 12 lessons. In this first unit, students are taught about disability in general. In the second unit, students are taught about Paralympic sports. When this intervention was first administered, the focus was on sledge hockey, but theoretically any parasport could be the focus sport. Lindsay and Edwards (2013) observed that six-session interventions have been sufficient for changing students' attitudes toward disability, but Leigers and Myers (2015) concluded that interventions tend to be better when they are longer in duration. In addition, Chae, Park, and Shin (2018) found that the number of sessions was more important than the total duration.

The intervention was set up as a spiral curriculum, which is a method for designing sequential lessons in such a way that the instructor meets students where they are and revisits concepts with increasing complexity (Bruner 196o; Harden 1999). The lessons were also developed with consideration of strategies for differentiated instruction (Tomlinson 2001) and meaningful student engagement (Valle \& Connor 2011). In addition to these general strategies, however, when administering these lessons, it is important for researchers and/or teachers to consider individual student's needs.

Within the TI students are given an opportunity to learn about a variety of concepts related to disability, interact with people who have disabilities, and reflect upon these experiences. Tindall (2013) describes three levels that can be achieved in educational interventions.

In Level I (exposure), students are introduced to different types of disability through various methods such as picture images, YouTube videos and other websites. In Level II (experience), students actually get to experience different types of disability (e.g. a blindfolded partner walk or spending time using a wheelchair). In Level III (ownership), students begin to advocate for persons with disabilities, taking it upon themselves to ensure that persons with disabilities are treated fairly and equally. (p. 459)

In this intervention students have an opportunity to work through all three levels. Disability simulations (i.e., Level II) has been criticized by scholars in this field of research (Cone \& Cone 2016; Flower, Burns, \& Bottsford-Miller 
2007; Silverman 2015). Nevertheless, these strategies can be effective when approached carefully and in conjunction with other intervention elements. Scholars recommend that if this method is going to be used, that the lived experiences of people with disabilities be included (Silverman 2015) and that ample time is taken to debrief these embodied experiences (Flower, Burns, \& Bottsford-Miller 2007).

The lesson sequence for each unit is: two cognitive lessons, two behavioural lessons, and two affective lessons. The cognitive lessons precede the behavioural lessons so students can learn about disability before they interact with people who have disabilities. It has been suggested that this strategy may help to reduce potential fears about contact experiences (Armstrong et al. 2016). The affective lessons come after the behavioural lessons, so the students can reflect upon the emotions they experience during the behavioural lessons. This lesson sequence is in line with experiential learning theory. Lewis and Williams (1994) explained that experiential learning 'immerses learners in an experience and then encourages reflection about the experience to develop new skills, new attitudes [emphasis added], or new ways of thinking' (p. 5).

Each lesson explicitly focuses on one of the three dimensions of attitude. The use of this strategy does not suggest the absence of the other dimensions during these lessons. For instance, social interactions with peers who have disabilities are categorized primarily in the behavioural dimension. This experience probably has impacts on both the affective and cognitive dimensions of attitude as well. This crossover works within the Tripartite Theory of attitude, as it is believed that these dimensions work together to determine attitude. The purpose of organizing the lessons in this way is to ensure that each dimension is given ample attention and time. The intention of the TI is to move beyond the status quo, which has been to focus on one or two dimensions of attitude, despite tripartite measurements. The hope is that this approach may help to address the complexity of students' attitudes toward disability. Furthermore, it would be useful if researchers were more explicit about the dimension of attitude within this field of research.

\section{Expert Panel}

This 12-lesson intervention was developed with consultation from the literature on students' attitudes toward disability and evidence-based pedagogy, which is cited below throughout the description of each lesson. To enhance the quality of the TI and ensure the appropriateness of each lesson, an expert panel also reviewed the lessons before the initial implementation. The lessons were sent out to fifteen community members. The members of the expert panel included four university professors, who conduct research in special education, social 
justice, and disability studies, one retired special educator, two educational assistants from different school boards, an elementary gym teacher, a school board speech-language pathologist, a special education teacher, a behaviour therapist, two sledge hockey players who have disabilities, and two junior-level educators.

All members of the expert panel were sent the intervention materials and a feedback form (see Appendix A). Expert panellists had one month to respond. All of the expert panel members responded except for one (i.e., 14 of the 15). The feedback received was taken into account and the lessons were updated where appropriate. For example, one of the teachers suggested students role-play the scenarios from lesson three. Each suggestion received was considered in reference to the intervention as a whole. Another expert suggested that the intervention include a culminating activity. While this suggestion may have enhanced elements of the intervention, it was ultimately not included in an effort to keep the lessons balanced (i.e., 2 cognitive-based, 2 behavioural-based, and 2 affective-based lessons in each unit).

\section{Unit \#1: What is Disability?}

The first unit consists of six lessons. Within these lessons, students are introduced to the concept of disability. The first two lessons target the cognitive dimension of attitude. Students develop their own definition of disability and draw a picture of a character who has a disability, discuss different types of disabilities, and explore the concept of inclusion. Lessons three and four focus on the behavioural dimension of attitude. In these lessons, students develop and role-play strategies for inclusion, and share a physical education period with students who have disabilities. Finally, lessons five and six provide students with an opportunity to consider their feelings about disability and reflect on their contact experience.

Lesson one (cognitive dimension). To begin the first lesson, students are presented with the word unique. Students are asked how many of them have heard this word before and if they know what the word unique means. To conclude this introductory discussion, students are asked to share something that makes them unique and to discuss the importance of differences between people. Alternatively, this opening activity can be flipped to become the closing activity for this lesson. This is recommended for researchers who are collecting the students' drawings and definitions as data to avoid priming the students' responses. Next, students are given a worksheet with the word disability on it and are responsible for writing what they think this word means. Once everyone has written a definition for the word disability, students are prompted to share their responses, which will facilitate a brief dialogue. Starting with what 
the students already knew and allowing them to build on that knowledge is in line with a constructivist learning approach. Santrock (2018) defines constructivism as 'a learner-centered approach to learning that emphasizes the importance of individuals actively constructing knowledge and understanding with guidance from the teacher' (p. G-2). Connor and Bejoian (2007) presented 20 techniques for teaching students about disability, two of which were utilized in this lesson. The two techniques used were: a) teaching students about differences, and $b$ ) studying the word disability.

Next, the second half of the worksheet asks students to draw a character with a disability and to describe their character's disability. Georgiadi and colleagues (2012) utilized a similar draw and write technique and pointed out that this is engaging and can facilitate students' thought processes regarding disability. The worksheet with the definition and drawing can be used as an assessment for teachers and/or as qualitative data for researchers (Freer 2020). The purpose of this activity is to discuss different types of disability, which can be illustrated using a concept map based on students' responses. Croasdell, Freeman, and Urbaczewski (2003) have pointed out that concept maps are a good way of visually representing students' existing knowledge on a topic.

Lesson two (cognitive dimension). After a review of the first lesson, students are asked to gather around for a reading of the book Keep Your Ear on the Ball (Genevieve \& Lyon 2009). This children's book tells the story of Davey's experience at a new school. The students in Davey's class are intrigued by his exceptionality (i.e., blind) and want to help him. Davey is sure to let them know that he can do things on his own. Davey and his classmates face a challenge on the kickball field but overcome it by coming up with an effective strategy for inclusion.

Several children's books were considered, but this picture book was ultimately selected with the help of a guide for choosing children's literature about disability (Artman-Meeker, Grant, \& Yang 2016). The book helps to challenge preconceived notions about disability because the main character showcases self-advocacy by clearly articulating what he is able to do and what he need help with.

After completing the book, students are prompted to discuss some of the main ideas with a think-pair-share (TPS) activity. TPS is an activity where students are first asked to work individually, then they are asked to pair up with a partner, and finally students engage in a class-wide discussion. TPS effectively helps to enhance student engagement (Kothiyal et al. 2013). Details on each TPS prompt can be found in the lesson materials.

Lesson three (behavioural dimension). This lesson begins with a review of important take-home messages from the story Keep Your Ear on the Ball (Genevieve \& Lyon 2009). In particular, this lesson builds off of the final TPS 
question from the previous lesson, which asks students to develop strategies for inclusion. Students are challenged to consider how they might practice inclusion in their own school, like the characters in the storybook. To follow, students are engaged in a brief discussion about the importance of including everyone, with an emphasis on the students' own behaviour. Scholars in the field of DSE have critiqued traditional special education's reliance on the medical model and have pushed for full inclusion (Baglieri et al. 2011; Valle \& Connor 2019). Some have even gone as far to say that full inclusion is a social justice issue (Artiles, Harris-Murri, \& Rostenberg 2006).

Next, students are divided into four groups. In these groups, the students read and discuss a brief scenario about a student who is being excluded for one reason or another. Not all of the cases focus specifically on the exclusion of a character with a disability, to emphasize that inclusion is about everyone and to avoid framing disability as an inherit other. The four scenarios include: two students with disabilities, a student who is shy, and student who is an English language learner. In small groups, students are asked to develop strategies on a piece of chart paper to better include the fictional student from the scenario. At regular intervals, students will rotate the chart paper to another group until each of the four groups has responded to all four scenarios. Once the chart paper is back to its original group, the students will select their favourite strategies to present to the class in a short role-play activity.

Lesson four (behavioural dimension). In the fourth lesson, students will be provided with an opportunity to interact with their peers who have disabilities in an inclusive physical education class. This can be facilitated with segregated classes within the school. If the school does not have separate classes for students with special education needs, one could work with support staff to bring students with exceptionalities from other classes together for this gym period. During this lesson, encourage students to build on the strategies for inclusion discussed in the previous lesson.

Students with and without disabilities will play cooperative games with one another. Dyson (2002) explained that cooperative learning is 'a dynamic instructional format that can teach diverse content to students at different grade levels. Students work together in structured, heterogeneous groups to master subject matter content. They are responsible ... for helping their group-mates learn' (p. 69). Additionally, it has been suggested that cooperative games help to enhance pro-social behaviour (Street et al. 2004). Playing cooperative games provides students with and without exceptionalities an opportunity to have positive interactions with one another.

Allport's (1954) contact theory suggests that contact can reduce prejudice against out-groups if the contact has: opportunity to experience equal status, 
common goals, intergroup cooperation, and authority support. The games are set up to achieve this, but teachers and support staff need to facilitate positive interactions for authority support. Contact between students with and without disabilities that intentionally meets all four of these criteria has shown to be effective in positively influencing children's attitudes toward disability (e.g., Zitomer \& Reid 2011). In addition, researchers who have conducted literature reviews on students' attitudes toward disability have suggested that providing students with opportunities to engage in social contact with people who have exceptionalities has been shown to be one of the most effective ways of enhancing students' attitudes toward disability (e.g., Freer 2020; Lindsay \& Edwards 2013; Macmillan et al. 2014).

Lesson five (affective dimension). This lesson starts by debriefing the cooperative gym class. Next, students are introduced to the six basic families of emotion (Ekman 1992). The lesson plan includes a teacher resource section for those who are not familiar with this theory. Other emotions have been hypothesized, but according to Ekman's theory, the six primary families of emotion include: anger, sadness, joy, surprise, disgust, and fear.

Students are provided with a stack of sticky notes and are asked to write one emotion they know on each note. Students are encouraged to write as many different emotions as they can. When they have generated several emotions, students may come up to the front and place their sticky notes under one of the six emotion categories written on the board or easel. Once the students place their notes on the board, the emotion words are to be read aloud and discussed as a class.

Next, students will complete a worksheet, in which they are asked to identify how they were feeling and how they thought their peers with exceptionalities were feeling during the inclusive gym class. The students are encouraged to use the list of emotions that they generated to help identify and explain their feelings. The sticky notes can then be used to create a worksheet with their different emotion words for a subsequent lesson (see teacher's note in the lesson plan). Worksheets may be collected for educational assessment and/or qualitative data.

Lesson six (affective dimension). In the final lesson of the first unit, students are given an opportunity to consider why people may feel certain emotions about disability. To begin this lesson, the families of emotions are reviewed. After a brief review, students are placed into groups of three to five. Each group receives a chart paper with six boxes, one labeled with each emotion family. In the first implementation of the TI, students were asked to consider why people might feel each of these six emotions regarding disability. Instead, some groups wrote why a person with a disability may feel each of 
these emotions. Based on the initial offering of the TI, this activity was modified to make the lesson more engaging and age appropriate. Now, students will be presented with fictional characters that experience each of the emotions when they hear about or meet a person with a disability. From there, the students will be asked to consider why they think the character in the scenario feels a particular way. To conclude this lesson, each group can be asked to share their contributions with the class. The intention of this closing discussion is to help students to critically analyze prejudicial feelings toward people with disabilities. The teacher's notes section of the lesson plan offers talking points that may help with facilitating the closing discussion.

\section{Unit \#2: Disability and Sports}

The second unit focuses on disability and sports and also contains six lessons. This unit follows the same lesson sequence as the previous unit (i.e., two cognitive, two behavioural, and two affective lessons). In lessons seven and eight, students are introduced to a variety of Paralympic sports. In lessons nine and ten, the students will be provided with an opportunity to play a parasport. Finally, in lessons 11 and 12, students reflect on their simulation experiencs and discuss the concept of empathy.

Researchers in this field continue to acknowledge parasports as a useful and effective strategy for teaching students about disability (Tindall 2013). Integrating Paralympic sports into intervention efforts can help bring awareness to parasports but can also help to blur the socially constructed lines between disability and ability, as the 'disabled' athletes showcase tremendous ability. Additionally, when able-bodied children play parasports, they can temporarily experience a disability simulation, while also developing an appreciation for the physical demands of parasports. When using Paralympic sports in educational interventions though, it is important to be aware of some criticisms. For example, Bellieni (2015) took issue with the Paralympics event itself by arguing that it ought to be part of the Olympics. He argued that the term Paralympics takes away from the fact that these professionals are displaying great feats of athleticism. Additionally, concerns have arisen with regard to the way in which disability has been presented during Paralympic coverage. Researchers and teachers need to be careful not to reinforce stereotypes when presenting Paralympic sports and athletes to students.

Media representations of Paralympic athletes have been criticized for being sparse and perpetuating stereotypical views of disability. For example, Goggin and Newell (2000) pointed out that when Paralympic athletes are represented in the media it is often from a lens of inspiration for overcoming their disability rather than simply a presentation of their tremendous athletic ability in 
its own right. Similarly, Silva and Howe (2012) pointed out the problems with presenting athletes as 'supercrips'. They explained,

Supercrip can be defined as a stereotype narrative displaying the plot of someone who has "to fight against his/her impairment" in order to overcome it and achieve unlikely "success." When uncritically interpreted, this type of narrative can be regarded as positive, contesting dominant views regarding disability as "negative" and "inferior." However, it is the negative "ethos" of disability that feeds the low expectations placed on the individual labeled as disabled in a way that any achievement is easily glorified, no matter how insignificant (Silva \& Howe 2012, pp. 178-179).

Within the TI, the skill and strength of the Paralympic athletes need to be emphasized in an effort to reshape the way in which students view ability and disability.

Lesson seven (cognitive dimension). To begin the second unit, students are asked to free-write everything they know about both the Olympics and Paralympics. Following this writing activity, students engage in a class discussion about their responses (see teacher's notes for talking points). To finish off this lesson, students are shown four (two-to-three-minute) YouTube videos. URLs can be found in the lesson materials. Each video showcases a different Paralympic sport so students can observe the sport in action as it is being described. These videos are directly from the official Paralympic Games YouTube channel to ensure copyright laws are not violated. The sports include: (1) goal ball, (2) wheelchair basketball, (3) sitting volleyball, and (4) Paralympic track and field events. Students are provided with an opportunity to respond verbally to each video. Researchers have found that teaching students about Paralympic sports helps to improve their attitudes toward disability (e.g., Evans, Bright, \& Brown 2015; Krahé \& Altwasser 2006; Tindall 2013).

Lesson eight (cognitive dimension). Lesson eight builds on the previous lesson's introduction to Paralympic sports. This lesson provides more detail on a focus parasport. When the TI was initially implemented, these lessons were created for sledge hockey. A researcher might choose a different parasport to focus on, depending on the equipment and volunteer athletes available in their community. The sledge hockey version is presented below.

To introduce sledge hockey, the first chapter of the book Fighting for Gold (Shultz Nicholson 2009) is read to the class. This short chapter introduces students to: the history of sledge hockey, some basic equipment, and rules of the game. Next, students are shown three (two-to-three-minute) YouTube videos from a Gatorade promo, featuring sledge hockey players in action. The videos 
are directly from Gatorade's official YouTube channel to ensure there was no copyright infringement. These videos provide students with an opportunity to view the sledge hockey in action. The first video features sledge hockey athletes playing with National Hockey League ( $\mathrm{NHL}$ ) players. The two subsequent videos are from the point of view of the NHL players and the sledge hockey players, respectively. Like lesson seven, the students are given an opportunity to respond verbally to each of the videos.

The second part of this lesson requires volunteer parasport athletes. The athletes introduce themselves, talk about their disability, and how they got involved in in the chosen parasport. Next, the athletes show the students some of the equipment, discuss the rules, and take questions from the students. Providing students with an opportunity to meet athletes who have disabilities has also been an aspect of successful interventions (e.g., Moore \& Nettelbeck 2013).

Lessons nine (behavioural dimension). In lesson nine, students are given an opportunity to learn how to play a parasport under the guidance and coaching of the volunteer parasport athletes. For sledge hockey, this can be achieved by either using roller sledge hockey kits or by playing scooter hockey in the gym. Before the training drills begin, the students need to stretch and warm up. It can be helpful to consult with the volunteer athletes in advance to develop the practice drills for this lesson.

After the safety considerations and rules are reviewed with the students, the athletes will model and coach the students on how to play their sport. Each athlete can take a small group of students in the gym and run through the drills. If shifts are required due to equipment restrictions, students who are not participating should be instructed to be good spectators by encouraging and cheering on their classmates.

Lessons 10 (behavioural dimension). In lesson 10, the students are given an opportunity to play a game of the selected parasport with the para athletes acting as the referees. After warming up, the class can be split into two teams. Safety and rules need to be reviewed with the students before the game begins. Safety is an important consideration for any parasport selected. In the initial implementation of the TI, the roller sledge hockey practice and games were completely non-contact and goalies were not permitted. In addition, safety guidelines from the Ontario Physical and Health Education Association (OPHEA 2018) were followed. Since there are no instructions for roller sledge hockey, the guidelines for scooter boards and floor ball were consulted. In addition, further precautions were taken for good measure. For example, boundaries were created with pylons away from the gym walls and benches to avoid collisions and the students wore protective glasses. In the 
future, researchers or teachers might consider adding helmets and hockey gloves. For all safety requirements, it is recommended that school administers be consulted.

Playing a parasport can give the students an opportunity to temporarily simulate the experience of having a disability. Using sports as a disability simulation task has also been an aspect of effective interventions (Krahé \& Altwasser 2006; Li, Wu, \& Ong 2014; Tindall 2013, etc.). Evans, Bright, and Brown (2015) suggested playing Paralympic sports might be effective in enhancing students' attitudes toward disability because it allows the participants to embody a disability experience. In addition, Krahé and Altwasser (2006) pointed out that, 'Sports activities contain a fun element and create an atmosphere in which initial inhibitions can be overcome' (p. 61). These experiential learning opportunities also include contact experiences, as the athletes have disabilities.

Lesson 11 (affective dimension). To begin lesson 11, students experiences playing a parasport are debriefed. Cone and Cone (2016) have questioned the ethic of disability simulation activities and have suggested that students should focus on ability rather than disability. As such, this debriefing should include a dialogue about both ability and disability. For example, if students discuss how difficult the parasport was, they can also be reminded of how skilled the guest athletes were.

Next, the students complete a worksheet that asks them to consider the emotions they experienced during the disability simulation activities and how they might feel if they had a disability. Student are provided with a list of the emotions they had developed in lesson five. Again, worksheets can be used for assessment and/or qualitative data. In the initial offering of the TI, students were placed into small groups and encouraged to articulate their understanding of the word empathy. The Grade 4 students struggled to provide a description of the word. Based on this experience, this activity has been modified for future offerings. Students will now be presented with some perspective-taking tasks, which will provide the students with an opportunity to reflect on how others might feel in different scenarios.

Lesson 12 (affective dimension). To start this lesson, students are asked if they are familiar with the word empathy and are provided with a basic definition (see lesson notes). Once the concept of empathy has been presented, a group discussion can be facilitated (see lesson plan for discussion notes). When discussing empathy and disability, it is important to avoid charity-based models of disability, deficit thinking, and pity. As such, the focus of this discussion should be on emotional perspective taking. Next, students are asked to write an example of empathy from their own experience. The facilitator 
will model this before asking students to share. If students cannot think of a personal example, they can develop a short fictional story of a character who experiences empathy.

For the next activity, students are shown an animation short entitled The Present (Frey \& Cavalcanti 2014). This video should be shown from the official YouTube channel of the animation creators. In their literature review, Lindsay and Edwards (2013) found that incorporating multimedia was associated with successful interventions. Students are prompted to analyze the video with questions about the character's emotions. The clip will be paused at key times (see lesson plan for details) and students will be asked to identify the main character's emotions and evidence for their assertion. Finally, students are asked if their understanding of the character's emotions changed with the twist at the end of the clip.

To conclude, students engage in a class discussion about the challenges of understanding another's emotions. This video analysis activity provides students with an opportunity to practice identifying emotions, motives, perspective taking, and empathy. Giving students an opportunity to practice empathy with a person or character with a disability may be a key factor that has been largely unexplored by researchers who have examined students' attitudes toward disability (Law et al. 2017). In fact, Armstrong and colleagues (2016) suggested, 'increasing empathy may improve children's attitudes to disability' (p. 879).

\section{Practical Considerations}

\section{Training}

Teachers need to be mindful of their own preconceptions and make efforts to not present stereotypical views of disability. Training is advisable for those interested in facilitating the TI. Please email the author to set up a workshop or webinar. Most teachers do not have a background in disability studies. To facilitate the TI effectively, educators may need to develop a greater understanding of DSE. Utilizing DSE approaches to break down attitudinal barriers has been suggested as an effective strategy (Connor \& Gabel 2013). A brief overview of the TI lessons is provided in Table 1.

\section{Planning}

The TI requires a great deal of planning in advance. Researchers coming into a class need to develop trust with the staff, parents, and students. One 


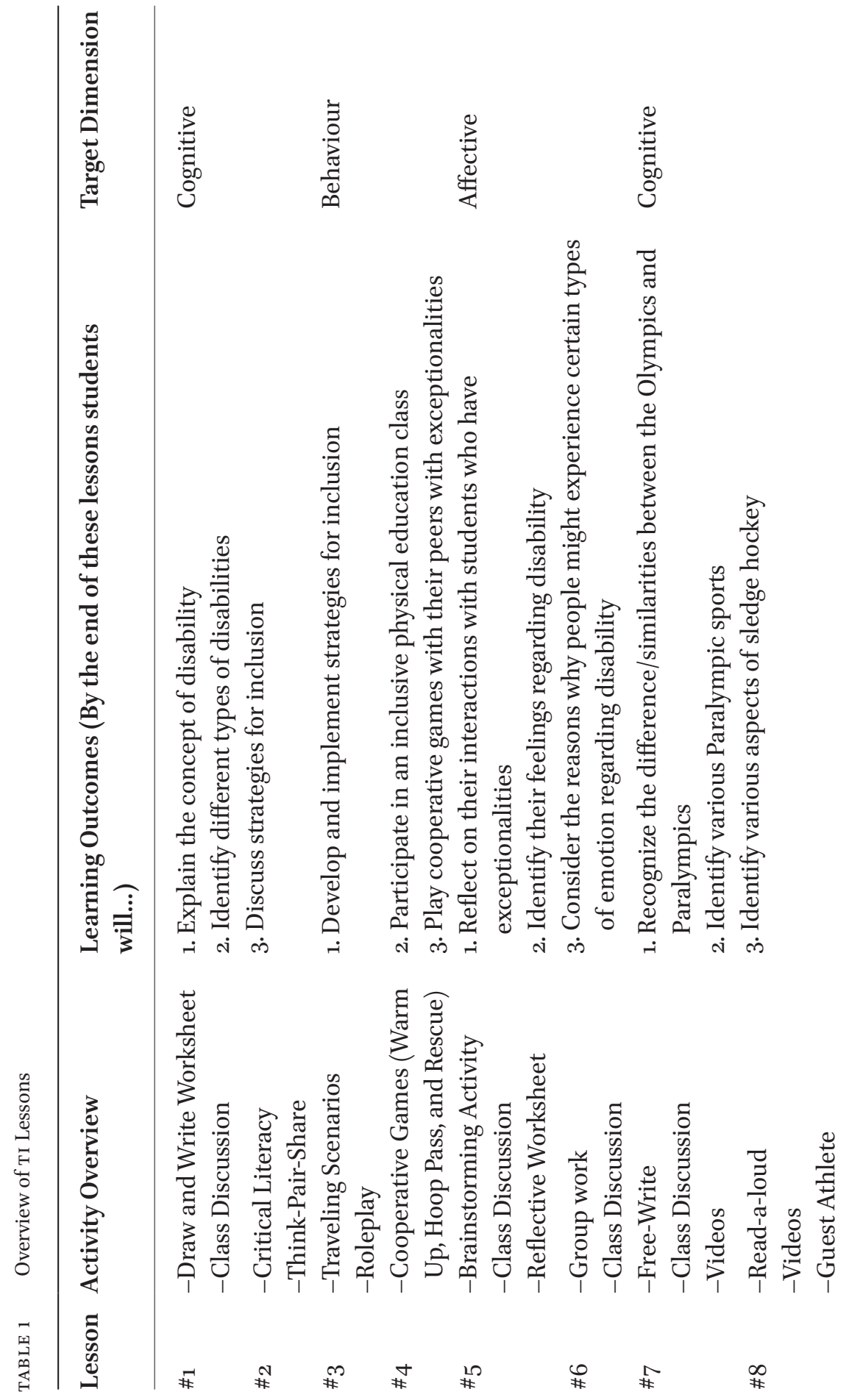




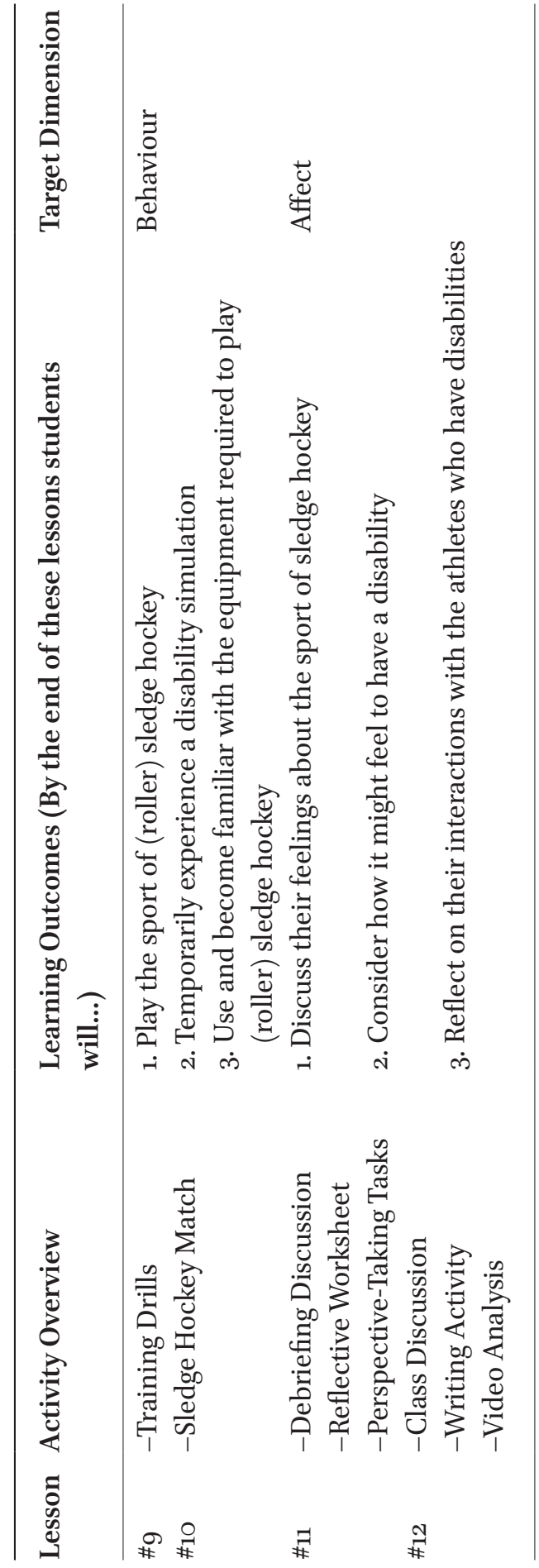


way to establish trust is to be transparent and flexible. School administrators and teachers involved should be provided with an opportunity to review and discuss each of the lessons. Once the lessons have been reviewed, school staff may have input (e.g., safety requirements in the gym, delivery schedule, scheduling the gym, etc.). It is recommended that the lessons be delivered at least two times a week. To acknowledge the complex nature of elementary schools, a flexible delivery plan is advised. For example, in the initial delivery of the TI, there were weeks when the teachers needed to reschedule (e.g., due to gym availability, school events, inclement weather, teacher illness, etc.).

In addition to meeting and collaborating with educators, it can be useful to establish a rapport with the students. A preliminary meeting with the class can assist in developing a better understanding of their day-to-day procedures and rules. See Appendix B for a discussion guide for establishing a positive learning environment. The guiding questions and prompts for this discussion had a Flesch-Kincaid readability score below a Grade 4 reading level.

Furthermore, researchers and teachers should be mindful of their school's culture/climate. For example, in an inclusionary setting, consideration should be given for the students who have exceptionalities in their classes. Efforts should be made to ensure these students do not feel centered out by the TI lessons. It is critically important that parents and administrators are informed about these lessons, especially since they are not a part of the mandatory curriculum.

Lessons four, eight, nine, and 10 require extensive planning because they involve collaboration with school and community partners. An important element of the TI is that it allows one to work alongside individuals with disabilities as allies to the intervention effort. In lesson four, it is recommended that facilitators draw from the school population to provide participants with authentic opportunities to experience inclusion. Another experience with people who have disabilities comes in unit two when the parasport athletes share their experiences, knowledge, and skills with the students. Stienstra and Wight-Felske (2003) pointed out the importance of oral history in research about disability by asserting that disability experiences should be shared from someone who has had that lived experience. It is recommended that any researcher or teacher thinking about integrating parasports into their classes first reach out to the athletes, coaches, and parents in their community, as they are experts within their respective areas.

\section{Time Commitment}

The 12 lessons of the TI take between 540 and 900 minutes to deliver. Parents and teachers may be concerned about time away from the required curriculum 
content. Teachers may need to look for cross-curricular connections to the lesson outcomes. While explicit connections to the curriculum have not been made, the TI lessons include elements of art, physical education, language arts, and social studies. Further examination of curriculum documents is necessary to determine the precise curricular outcomes at each grade level within junior education.

If educators are concerned about the time commitment of the TI, teachers might also select aspects of the intervention without completing all 12 lessons with their class. As a caution, the effect of the individual lessons or units has not been assessed. These efforts could be as simple as selecting a book to read with disability experiences present or as complex as working with a local parasport team to join existing health and physical education classes. Based on the findings of her study, Nowicki (2007) found that in general 'children need to be better informed [about disability]' (p. 418) and approaches such as these could help to bridge that gap. Educators have a social responsibility to teach students about diverse experiences, including disability.

\section{Intervention Fidelity}

Finally, researchers or teachers looking to adopt or adapt the TI for their use, should consider intervention fidelity. Murphy and Gutman (2012) identified attendance tracking as one important measure of a new intervention's fidelity. For a simple attendance tracking sheet, please see Appendix C. If the research taking place involves the randomization of participants, then an intention to treat (ITT) method ought to be used. Gupta (2011) explains that, 'ITT analysis includes every subject who is randomized according to randomized treatment assignment. It ignores noncompliance, protocol deviations, withdrawal, and anything that happens after randomization' (p. 109). If, however, researchers are working in already established classes that have not been selected randomly, a per-protocol analysis is recommended. 'Per-protocol [analysis] ... is defined as a subset of the ITT population who completed the study without any major protocol violations' (Gupta 2011, p. 109). Hernán and Robins (2017) further explain, 'When ... perfect adherence is unrealistic, one may redefine the per-protocol effect as the effect under 'satisfactory adherence' to the protocol (e.g., $8 \circ \%$ ), rather than the effect under $100 \%$ adherence to the protocol' (p. 1397).

\section{Conclusion}

Students' with disabilities continue to face attitudinal barriers at school. When students' peers have poor attitudes toward disability, this can negatively 
impact friendship development between students with and without disabilities. While educational interventions show promise for improving students' attitudes toward disability, most do not explicitly target affective, behavioural, and cognitive dimensions of attitude. The TI intentionally targets all three dimensions of attitude and utilizes evidence-based strategies. This intervention could be useful to teachers and researchers who are interested in teaching students about disability experiences. The TI has the potential to be a practical tool for promoting social inclusion and challenging ableism in elementary schools.

\section{References}

Allport, G. W. (1954). The nature of prejudice. Oxford, England: Addison-Wesley.

Armstrong, M., Morris, C., Abraham, C., Ukoumunne, O. C., and Tarrant, M. (2016). Children's contact with people with disabilities and their attitudes towards disability: A cross-sectional study. Disability and Rehabilitation, 38(9): 1-10. doi: 10.3109/og638288.2015.1074727.

Artiles, A. J., Harris-Murri, N., and Rostenberg, D. (2006). Inclusion as social justice: Critical notes on discourses, assumptions, and the road ahead. Theory into Practice, 45: 26o-268. doi: 10.1207/s15430421tip45o3_8.

Artman-Meeker, K., Grant, T. O., and Yang, X. (2016). By the book: Using literature to discuss disability with children and teens. TEACHING Exceptional Children, 48(3): 151-158. doi: 10.1177/0040059915618195.

Avramidis, E. (2013). Self-concept, social position and social participation of pupils with SEN in mainstream primary schools. Research Papers in Education, 28(4): 421442. doi:10.108o/o2671522.2012.673006.

Baglieri, S., Valle, J. W., Connor, D. J., and Gallagher, D. J. (2011). Disability studies in education: The need for a plurality of perspectives on disability. Remedial and Special Education, 32(4): 267-278. doi: 10.1177/0741932510362200.

Bellieni, C. (2015). Paralympics should be integrated into main Olympic games. Sport, Ethics and Philosophy, 9(1): 75-82. doi: 10.108o/17511321.2015.1041149.

Bruner, J. S. (1960). The process of education. Oxford, England: Harvard University Press. Chae, S., Park, E. Y., Shin, M. (2018). School-based interventions for improving disability awareness and attitudes towards disability of students without disabilities: A metaanalysis. International Journal of Disability, Development, and Education, 66(4):343362. doi: 10.1080/1034912X.2018.1439572.

Cone, T. P., and Cone, S. L. (2016). An alternative perspective on simulations. Strategies: A Journal for Physical and Sport Educators, 29(5):56. Retrieved from https://doi.org/ 10.108o/08924562.2016.1213064. 
Connor, D. J., and Bejoian, L. (2007). Cripping school curricula: 20 ways to re-teach disability. The Review of Disability Studies: An International Journal, 3(3): 1-11. Retrieved from http://www.rdsjournal.org/index.php/journal/article/view/285/885.

Connor, D. J., and Gabel, S. L. (2013). "Cripping" the curriculum through academic activism: Working toward increasing global exchanges to reframe (dis) ability and education. Equity \& Excellence in Education, 46(1): 100-118. doi: 10.1080/10665684.2013.750186.

Croasdell, D. T., Freeman, L. A., and Urbaczewski, A. (2003). Concept maps for teaching and assessment. Communications of the Association for Information Systems, 12: 396-405. Retrieved from http://aisel.aisnet.org/cais/volı2/iss1/24/.

de Boer, A., Pijl, S. J., and Minnaert, A. (2012). Students' attitudes towards peers with disabilities: A review of the literature. InternationalJournal of Disability, Development and Education, 59(4): 379-392. doi: 10.1080/1034912X.2012.723944.

de Boer, A., Pijl, S. J., Post, W., and Minnaert, A. (2012). Which variables relate to the attitudes of teachers, parents and peers towards students with special educational needs in regular education? Educational Studies, 38(4): 433-448. doi: 10.1080/03055698.2011.643109.

de Boer, A., Pijl, S. J., Post, W., and Minnaert, A. (2013). Peer acceptance and friendships of students with disabilities in general education: The role of child, peer, and classroom variables. Social Development, 22: 831-844. doi: 10.1111/j.1467- 9507.2012.00670.x.

Dyson, B. (2002). The implementation of cooperative learning in an elementary physical education program. Journal Of Teaching In PhysicalEducation, 22(1): 69-85. Retrieved from https://doi.org/10.1123/jtpe.22.1.69.

Ekman, P. (1992). An argument for basic emotions. Cognition \& Emotion, 6(3-4): 16920o. doi:10.1080/02699939208411068.

Evans, B. A., Bright, L. J., and Brown, J. L. (2015). Non-disabled secondary school children's lived experiences of a wheelchair basketball programme delivered in the east of England. Sport, Education and Society, 20(6): 741-761. doi: 10.1080/13573322.2013.808620.

Flower, A., Burns, M. K., and Bottsford-Miller, N. A. (2007). Meta-analysis of disability simulation research. Remedial and Special Education, 28(2): 72-79. doi: 10.1177/07419325070280020601.

Freer, J. (2020). Students' attitudes toward disability: A tripartite intervention. Doctoral Dissertation. University of Windsor. Ontario, Canada.

Frey J. and Cavalcanti, F. (2014). The Present [animation short]. Filmakademie BadenWuerttemberg (Institute for Animation and VFX).

Genevieve, P., and Lyon, L. (Illustrator). (2009). Keep Your Ear on the Ball. Gardiner, Maine: Tilbury House.

Georgiadi, M., Kalyva, E., Kourkoutas, E., and Tsakiris, V. (2012). Young children's attitudes toward peers with intellectual disabilities: Effect of the type of school. 
Journal of Applied Research in Intellectual Disabilities, 25(6): 531-541. doi: 10.1111/j.1468-3148.2012.00699.x.

Giagazoglou, P. and Papadaniil, M. (2018). Effects of a storytelling program with drama techniques to understand and accept intellectual disability in students $6-7$ years old. A pilot study. Advances in Physical Education, 8(2): 224-237. doi: 10.4236/ ape.2018.82020.

Godeau, E., Vignes, C., Sentenac, M., Ehlinger, V., Navarro, F., Grandjean, H., and Arnaud, C. (2010). Improving attitudes towards children with disabilities in a school context: A cluster randomized intervention study. Developmental Medicine \& Child Neurology, 52(10): e236-e242. doi: 10.1111/j.1469-8749.2010.03731.

Goggin, G. and Newell, C. (2000). Crippling Paralympics? Media, disability, and olympism. Media International Australia, Incorporating Culture \& Policy, 97: 71-83. doi: 10.1177/1329878Xooo970o110.

Gupta, S. K. (2011). Intention to treat concept: A review. Perspectives of Clinical Research, 2(3): 109-112. doi: 10.4103/2229-3485.83221.

Harden, R. M. (1999). What is a spiral curriculum? Medical Teacher 21(2): 141-143. doi: $10.1080 / 0142159997975^{2}$.

Hehir, T. (2002). Eliminating ableism in education. Harvard Educational Review, 72(1): 1-32. doi: 10.17763/haer.72.1.03866528702g2105.

Hernán, M. A. and Robins, J. M. (2017). Pre-protocol analyses of pragmatic trials. New England Journal of Medicine, 377(14): 1391-1398. doi: 10.1056/NEJMsm1605385.

Jackson, L. A., Hodge, C. N., Gerard, D. A., Ingram, J. M., Ervin, K. S., and Sheppard, L. A. (1996). Cognition, affect, and behavior in the prediction of group attitudes. Personality and Social Psychology Bulletin, 22(3): 306-316. doi:10.1177/01461672962230o9.

Jones, S. R. (1996). Toward inclusive theory: Disability as social construction. NASPA Journal, 33(4):347-354. doi: 10.1080/oo220973.1996.11072421.

Koster, A., Pijl, S. J., Nakken, H., and Van Houten, E. (2010). Social participation of students with special needs in regular primary education in the Netherlands. International Journal of Disability, Development and Education, 57(1): 59-75. doi: 10.1080/10349120903537905.

Kothiyal, A., Majumdar, R., Murthy, S., and Iyer, S. (2013). Effect of think-pair-share in a large CS1 class: $83 \%$ sustained engagement. Proceedings of the 9 th Annual acm Conference on International Computing Education Research. (icer): 137-144. doi: $10.1145 / 2493394.2493408$.

Krahé, B., and Altwasser, C. (2006). Changing negative attitudes towards persons with physical disabilities: An experimental intervention. Journal of Community \& Applied Social Psychology, 16(1): 59-69. doi:10.1002/casp.849.

Law, Y., Lam S., Law, W., and Tam Z. Y. W. (2017). Enhancing peer acceptance of children with learning difficulties: classroom goal orientation and effects of a storytelling 
programme with drama techniques. Educational Psychology, 37(5): 1-13. doi: 10.1080/01443410.2016.1214685.

Leigers, K. L., and Myers, C. T. (2015). Effect of duration of peer awareness education on attitudes toward students with disabilities: A systematic review. Journal of Occupational Therapy, Schools \& Early Intervention, 8(1): 79-96. doi: 10.1080/ 19411243.2015.1021067.

Lewis, L.H. and Williams, C.J. (1994). Experiential learning: Past and present. In , L. Jackson and , R.S. Caffarella (Eds.), Experiential Learning: A New Approach (pp. 516). San Francisco: Jossey-Bass.

Li, C., Wu, Y., and Ong, Q. (2014). Enhancing attitudes of college students towards people with intellectual disabilities through a coursework intervention. Journal of Developmental and Physical Disabilities, 26(6): 793-803. doi: 10.1007/ s10882-014-9395-z.

Lindsay, S., and Edwards, A. (2013). A systematic review of disability awareness interventions for children and youth. Disability and Rehabilitation, 35(8): 623- 646. doi:10.3109/o9638288.2012.70285o.

Lloyd, A., Smith, M., Dempsey, I., Fischetti, J., and Amos, K. (2017). Short- and mediumterm of Just Like You disability awareness program: A quasi-experimental comparison of alternative forms of program delivery in New South Wales' primary schools. Australian Journal of Education, 61(3): 288-304. doi: 10.1177/0o04944117730649.

Louari, M. (2013) Can typical students define the word "disability"? A pilot study about their knowledge or misconceptions. Procedia Social and Behavioral Sciences 93: 699-703. doi: 10.1016/j.sbspro.2013.09.264.

Macmillan, M., Tarrant, M., Abraham, C., and Morris, C. (2014). The association between children's contact with people with disabilities and their attitudes towards disability: A systematic review. Developmental Medicine and Child Neurology, 56(6): 529-546. doi: 10.1111/dmcn.12326.

Moore, D., and Nettelbeck, T. (2013). Effects of short-term disability awareness training on attitudes of adolescent schoolboys toward persons with a disability. Journal of Intellectual \& Developmental Disability, 38(3): 223-231. doi: 10.3109/13668250.2013.790532.

Murphy, S. L., and Gutman, S. A. (2012). Intervention fidelity: A necessary aspect of intervention effectiveness studies. The American Journal of Occupational Therapy, 66(4): 387-388. doi: 10.5014/ajot.2010.005405.

Nowicki, E. A. (2007). Children's beliefs about learning and physical difficulties. International Journal of Disability, Development and Education, 54,(4): 417-428. doi: 10.108 o/10349120701654647.

Nowicki, E. A., and Sandieson, R. (2002). A meta-analysis of school-age children's attitudes towards persons with physical or intellectual disabilities. International 
Journal of Disability, Development and Education, 49(3): 243-265. doi: 10.1080/ 1034912022000007270.

Oliver, M. (1996). Understanding disability: From theory to practice. New York City, NY: Macmillan.

Ontario Physical and Health Education Association (opheA). (2018). In Ontario Physical Education Safety Requirements: Elementary. Retrieved from http://safety. ophea.net/elementary.

Petry, K. (2018). The relationship between class attitudes towards peers with a disability and peer acceptance, friendship and peer interactions of students with a disability in regular secondary schools. European Journal of Special Needs Education, 33(2): 254-268. doi: 10.108o/08856257.2018.1424782.

Rillotta, F., and Nettelbeck, T. (2007). Effects of an awareness program on attitudes of students without an intellectual disability towards persons with an intellectual disability. Journal of Intellectual \& Developmental Disability, 32(1): 19-27. doi: 10.1080/13668250701194042.

Rosenbaum, P. L., Armstrong, R. W., and King, S. M. (1986). Children's attitudes toward disabled peers: A self-report measure. Journal of Pediatric Psychology, 11(4): 517-530. doi: 10.1093/jpepsy/11.4.517.

Santrock, J. W. (2018). Educational Psychology (6th ed.) New York City, NY: McGraw Hill.

Shapiro, J. P. (1994). No pity: People with disabilities forging a new civil rights movement. New York City, NY: Three Rivers.

Shultz Nicholson, L. (2009). Fighting for gold: The story of Canada's sledge hockey Paralympic gold. Toronto, Ontario: Lorimer.

Silva, C. F. and Howe, P. D. (2012). The (in)validity of supercrip representation of Paralympian athletes. Journal of Sport and Social Issues, 36(2): 174-194. doi: $10.1177 / 0193723511433865$.

Silverman, A. M. (2015). The perils of playing blind: Problems with blindness simulation and a better way to teach about blindness. Journal of Blindness Innovation \& Research, 5(2). doi: 10.5241/5-79.

Simplican, S. C., Leader, G., and Kosciulek, J. (2015). Defining social inclusion of people with intellectual and developmental disabilities: An ecological model of social networks and community participation. Research in Developmental Disabilities, 38: 18-29. doi: http://dx.doi.org/10.1016/j.ridd.2014.10.oo8.

Slee, R., Corcoran, T., Best, M. (2019). Disability Studies in Education-Building platforms to reclaim disability and recignise disablement. Journal of Disability Studies in Education 1(1): 1-11. doi: 10.1163/25888803-00101002.

Stienstra, D. and Wight-Felske, A. (2003). Making equality: History of advocacy and persons with disabilities in Canada. Concord, Ontario: Captus. 
Storey, K. (2007). Combating ableism in schools. Preventing School Failure, 52(1): 56- $5^{8}$. doi: 10.3200/PSFL.52.1.56-58.

Street, H., Hoppe, D., Kingsbury, D., and Ma, T. (2004). The game factory: Using cooperative games to promote pro-social behaviour among children. Australian Journal of Educational \& Developmental Psychology, 4: 97-109. Retrieved from https://eric.ed.gov/?id=EJ815556.

Tavares, W. (2011). An evaluation of the Kids Are Kids disability awareness program: Increasing social inclusion among children with physical disabilities. Journal of Social Work in Disability \& Rehabilitation, 10(1): 25-35. doi:10.1080/15367 10X.2011.546296.

Tindall, D. (2013). Creating disability awareness through sport: Exploring the participation, attitudes and perceptions of post-primary female students in Ireland. Irish Educational Studies, 32(4): 457-475. doi: 10.108o/o3323315.2013.859339.

Tomlinson, C. A. (2001). How to differentiate instruction in mixed ability classrooms. (2nd ed.). Alexandria, Virginia: ASCD.

Valle, J. W., and Connor, D. J. (2011). Rethinking disability: A disability studies approach to inclusive practices. New York City, NY: McGraw-Hill.

Valle, J. W., and Connor, D. J. (2019). Rethinking disability: A disability studies approach to inclusive practices. (2nd ed.). New York City, NY: McGraw-Hill.

Vignes, C., Coley, N., Grandjean, H., Godeau, E., and Arnaud, C. (2008). Measuring children's attitudes towards peers with disabilities: A review of instruments. Developmental Medicine \& Child Neurology, 5O(3): 182-189. doi: 10.1111/j.1469-8749.2008.02032.x.

Vignes, C., Godeau, E., Sentenac, M., Coley, N., Navarro, F., Grandjean, H., and Arnaud, C. (2009). Determinants of students' attitudes towards peers with disabilities. Developmental Medicine \& Child Neurology, 51(6): 473-479. doi: 10.1111/j.1469-8749.2009.03283.x.

World Health Organization (wHo). (2O2O). In Health topics. Retrieved from http:// www.who.int/topics/disabilities/en.

Zitomer, M. R., and Reid, G. (2011). To be or not to be-able to dance: Integrated dance and children's perceptions of dance ability and disability. Research in Dance Education, 12(2): 137-156. doi: 10.1080/14647893.2011.575224. 


\section{Appendix A}

Expert Panel Feedback Form

Expert Panel Checklist

Name:

Organization:

Position:

*The above information is only for my own organization and will not be presented in the final report. I will state generally that the expert panel was made up of [insert professions] from school boards, higher education institutes, etc.

Lesson Materials:

Suggestions:

Lesson Plan \#1

Worksheet 1: What is Disability?

Lesson Plan \#2

Lesson Plan \#3

Lesson Plan \#4

Lesson Plan \#5

Worksheet \#2: Cooperative Games Reflection

Lesson Plan \#6

Lesson Plan \#7

Lesson Plan \#8

Lesson Plan \#9

Lesson Plan \#10

Lesson Plan \#11

Worksheet \#3: Sledge Hockey Reflection

Lesson Plan \#12 


\section{Appendix B}

Discussion Guide: Establishing a Positive Learning Environment

Guiding Questions:

I will be coming to your class for the next few weeks. Can you tell me what class rules you have? Prompt: What happens if someone is not following the rules? [If not identified, discuss: hand raising, washroom policy, what happens when someone is away, etc.]

In my classes, I have lots activities. What can we do so everyone can be involved? Prompt: How can we show our classmates support? [If not identified, discuss: that everyone's perspective is valued, there are no bad questions or answers, etc.]

What can you do if you feel uncomfortable during one of the lessons? Prompt: What could you if you didn't want to join in? [If not identified, discuss: tell the teacher, the researcher, or their parents.]

\section{Appendix C}

Attendance Tracker Form

Lesson/Date Students Absent Other Notes Lesson Duration

Lesson 1:

Lesson 2:

Lesson 3:

Lesson 4:

Lesson 5:

Lesson 6:

Lesson 7:

Lesson 8:

Lesson 9:

Lesson 10:

Lesson 11:

Lesson 12: 